\title{
AGN in the Atacama Cosmology Telescope Survey Data
}

\author{
Megan Gralla, T. Marriage and ACT Collaboration
}

Johns Hopkins University, USA

E-mail: mgralla@pha.jhu.edu

\begin{abstract}
The Atacama Cosmology Telescope (ACT) is a six-meter telescope in the Atacama Desert that observes simultaneously in frequency bands centered at 150, 220 and $270 \mathrm{GHz}$. $\mathrm{ACT}$ has surveyed an area of approximately 450 square degrees in the south $(-55 \mathrm{deg})$ and approximately 500 square degrees along the equator. Most of the sources detected by ACT are blazars. I will present source counts and spectral indices as well as multi-wavelength properties determined by matching with external datasets. In addition to studying sources detected in the survey, we have used the ACT data to statistically study the millimeter properties of radio sources selected from low frequency $(1.4 \mathrm{GHz})$ surveys.
\end{abstract}

